\title{
Research on 3G Technologies-Based Agricultural Information Resource Integration and Service
}

\author{
Nengfu Xie ${ }^{1,3, *}$ and Wensheng Wang ${ }^{2}$ \\ ${ }^{1}$ Agricultural Information Institute, The Chinese Academy of Agricultural Sciences, \\ Beijing, China, 100081 \\ nf.xieg@caas.net.cn \\ ${ }^{2}$ Key Laboratory of Digital Agricultural Early-warning Technology, Ministry of Agriculture, \\ The People's Republic of China \\ ${ }^{3}$ Agricultural Information Institute, No.12 Zhongguancun South St., \\ Haidian District Beijing 100081, P.R. China \\ Tel.: +86-10-82109819; Fax: +86-10-82109819
}

\begin{abstract}
With the urgent demand of agricultural information for farmers and quick development of telecommunications industry in china, the convenience, quickness and validity of agricultural information service are becoming more important. Now, the problem of the digital divide between rural and urban areas comes from the shortage of effective information transmission means. But the agricultural information services limited by network bandwidth and geography will be changed completely with the mobile phone network coverage to rural areas, especially TD-SCDMA network into rural areas in china. The paper will introduce the development of the $3 \mathrm{G}$ technologies-based effect on the agriculture informationization, and discuss the problems of agricultural information resource integration and service adapted to $3 \mathrm{G}$ technologies in detail, based on which a $3 \mathrm{G}$ technologies-based agricultural information resource application mobile service hierarchy (3GAIMSH) is provided.
\end{abstract}

Keywords: Agricultural Information, 3G, TD-SCDMA, service design, integration.

\section{Introduction}

With the urgent demand of agricultural information for farmers and quick development of telecommunications industry in china, the convenience, quickness and validity of agricultural information service are becoming more important. The shortage of agricultural information, poor telecommunications networks and uncoordinated information resources are crucial problems facing agricultural information sources in china. How to integrate the agricultural information coming from different agricultural information sources and provide information service for farmers in rural areas, especially in many poor rural areas is the current research focus of information service because human factors are still the main means of collecting and disseminating

\footnotetext{
* Corresponding author.
} 
information in many rural areas. In the near future, wireless networks will appear with greater ubiquity, from public access networks offering connectivity in agricultural information services, to telecoms operated $3 \mathrm{G}$ and $4 \mathrm{G}$ networks, especially $\mathrm{TD}$ SCDMA network into rural areas in china, which allows 9 hundred million of farmers access to services as important and varied as information acquisition, health care, education, and financial and governmental services. The mobile phone has been described as the most likely modern digital device to support economic development in developing nations (The real digital divide, 2005). Tapan presented CAM - a framework for developing and deploying mobile applications in the rural developing world. Supporting minimal, paper-based navigation, a simple scripted programming model and offline multimedia interaction, CAM is uniquely adapted to rural user, application and infrastructure constraints (Parikh etc., 2006). The agricultural information services limited by network bandwidth and geography will be changed completely with the mobile phone network coverage to rural area, which will help solve the problem of "last kilometer" in agricultural informationization.

Third generation mobile network (3G) is the latest advancement in the field of mobile technology. Providing high bandwidth communication of $8 \mathrm{kbit} / \mathrm{s}-2 \mathrm{Mbit} / \mathrm{s}$ and a revolutionary introduction of multimedia services over mobile communication, it aims to make mobile devices into versatile mobile user terminals. TD-CDMA (Time Division CDMA) wireless technology in china can meet the rapidly growing demand for mobile broadband services in agricultural information systems. The mobile information services will make it easier and quicker to provide agricultural information services in today's more complex web environment.

Now, the problem of the digital divide between rural and urban areas comes from the shortage of effective information transmission means. But the agricultural information services limited by network bandwidth and geography will be changed completely with the mobile phone network coverage to rural areas, especially TDSCDMA network into rural areas in china. The paper will introduce the development of the $3 \mathrm{G}$ technologies-based effect on the agriculture informationization, and discuss the problems of agricultural information resource integration and service adapted to $3 \mathrm{G}$ technologies in detail, based on which a $3 \mathrm{G}$ technologies-based agricultural information resource application mobile service hierarchy is provided.

\section{The Problems of Agriculture Information Services}

As the amount and complexity of web agricultural information continues to grow, quick and efficient information retrieval \& intelligent information services are becoming a critical focus for research and development, especially for multiple heterogeneous information sources. The information sources are distributed at different locations, most of which are belonged to many research or commercial unites' private proprietary information. Such this situation makes public users or farms impossible to access the information. On the other hand, the usage rate of the distributed information is very low. In fact, these sources can be combined and integrated into a unified environment, which provides a united interface for users, requiring information, who will not need to reach each information and seeks the information that they need. 


\subsection{Digital Divide}

Currently, many information resources in a certain unit can not be very limited or no access at all. Though, Accessing the Internet will bring a wealth of information to all agriculture farmers in rural areas and will help in overcoming the digital divide. As most farmers in china have no hands-on experience or access to digital networks, and most information on the web is very confused that may bring many problems in agriculture production process and agriculture production market. On the other hand, most useful and practical information are coming from agriculture government and Research Institutes. But the access of such information may be limited. So we an open and integrated agricultural information system should be encouraged, which help farmers access a lot of useful information if each country tries to develop contents in the language people are using.

\subsection{Lack of Rapid and Timely Information Distribution}

With special characteristic such as region, domain and timeness, information required cannot reach users in many rural areas. Rural areas have spatially intermittent connectivity. By requiring online usage of an application, villagers would have to travel to a connected location to access it. This can be very inconvenient, possibly taking an entire day. One of the most innovative new tools for rural development might be in your pocket.

Over the last few years cell phone use has exploded in china, with more uses than anyone could have imagined. Using communication technologies for agricultural development was the theme of the World Conference on Agricultural Information and IT held in Japan last week. Over a billion people use the internet, but more than three times as many use cell phones. Especially with the 3D technology development, agricultural information required is no longer a problem for the most isolated rural area, but the big need is for good content.

\subsection{Limitations of Current Mobile Software Platforms}

While mobile phone hardware is well-suited to rural conditions, the same is not true for software. Mobile web interfaces are notoriously difficult to use, even for developed world users (George etc., 2001). Typing URLs with a numeric keypad is slow and painful. Therefore, users must rely on a portal or set of bookmarks to access web sites. Most web pages are designed for large screen resolutions, making navigation within a page also problematic (Parikh \& Lazowska, 2006). Currently mobile software platforms in agricultural domain have been established in many rural areas in China. However, how to push the right content to users in a right way is still a big problem because there is not a content-abundant integrated environment that has many applications and tools suitable $3 \mathrm{G}$ technology-based information process and distribution. Especially, with the emergence of $3 \mathrm{G}$ technology, information access and distribution are becoming very easily, and available for users in many rural areas.

\section{The 3GAIMSH Hierarchy}

At present, the mobile service of agricultural information had been conducted in many provinces in China, especially TD-SCDMA based network into rural areas in china 
recently. 3G mobile technology will make video telephony or video call in areas within a network coverage and use a wider range of value added services. Such mutimedia-base content will help farmers to understand agricultural information more easily. By $3 \mathrm{G}$ mobile network, the agricultural production direct, news, maket information and etc. will be transmitted to farmer's 3G mobile phones, which are available for viewing and exporting retrieved information.

In 3GAIMSH hierarchy, we provide a environment that integrate related different agricultural information sources into the agricultural information center by data adapter. The source can be deleted, added and indexed dynamatically. The center will also provide Update Tool guarantees that all data are always upto- date. The 3G-based information distribution service ensures that the information requested will be pushed farmer's 3G mobile phones quickly.

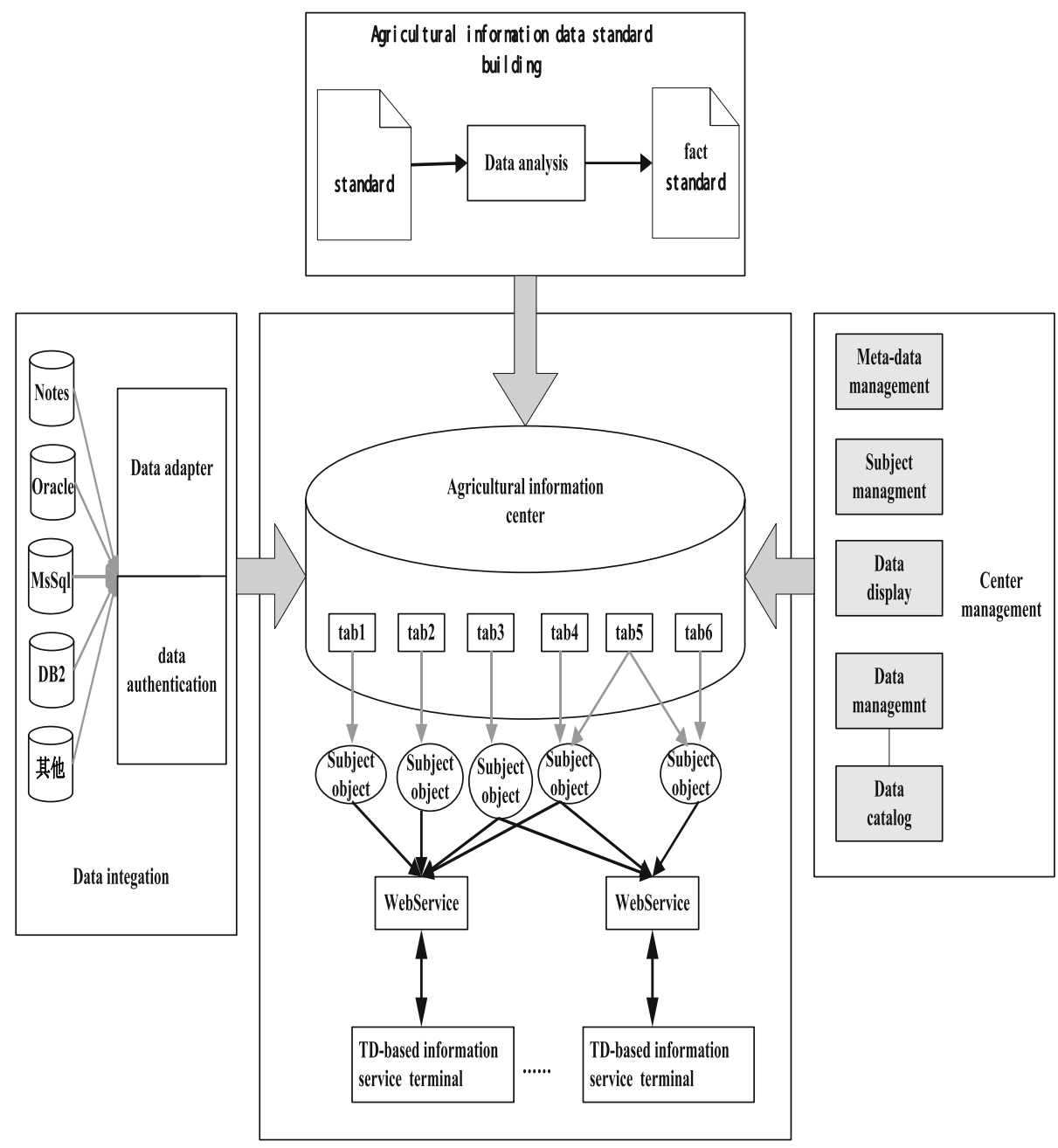

Fig. 1. The 3GAIMSH hierarchy 


\subsection{Agricultural Information Sharing Standard Building}

In order to let distributed information sources join the information center, the related agricultural information sharing standard must be built, including access standard, service standard, and exchange standard. In building these standards, firstly agricultural information model must be studied and get an application-suitable information standard. In the access standard, the criterion of information access interface must be defined. We have created a web service interface supporting the construction of distributed agricultural access interfaces. For information exchange, we have defined XML-based agriculture domain information exchange standard. To improve interoperability of Web Services, service standard will consider SOAP 1.1, UDDI 2, ... to help the deployment of profile compliant Web Services.

\subsection{Data Adapter}

Data adapter is an important part of the 3GAIMSH hierarchy. Data adapter provides the communication between the distributed sources and the agricultural information center. We can use the data adapter in combination with the web service-based interface. That is these two interfaces combine to enable both data access and data manipulation capabilities using access standard.

The data adapter can perform select, insert, update, delete and conversion information operations in the distributed information sources. For the insert, update and delete information operations, we are using the continuation of the select command perform by the data adapter. That is the data adapter uses the select statements to fill an information Set and use the other three commands (insert, update, delete) to transmit changes back to the distributed sources' database. In order to let the information into the agricultural information center, the information must be transferred according to exchange standard.

\subsection{Data Authentication}

Data authentication is important for many applications in data integration. The data authentication will keep in the agricultural information center the part of information source register $\&$ access data that can be handled by authentication module. The data authentication module can provide agricultural information center data from other sources, and implement an interface to create new information sources, which will be sharing for users. This also ensure that the data shared by a certain users with a given privilege.

\subsection{Data Management}

Data Management module is designed for the data add, delete, and query in distributed agricultural information integration environment. It will control the data quality of different databases, data service, and data clustering. It provides the management on the distributed information integration environment for helping administrator to regulate the basic management of agricultural information in the information center. 


\subsection{Metadata-Based Information Category and Service}

Metadata information category \& service will help users find the information they required easily. The information meta-data is building an object-oriented repository technology that is integrated with agricultural meta data management that process metadata, which will provide a united category that organize the information in information center, and a standard of the information' store, and support exchange of model data. The metadata-based information service will provide a united interface for users to access the information by metadata-based information category.

\subsection{Agricultural Information Distribution}

Agricultural information distribution is about assuring that the right agricultural information is available to the right agricultural users. In order to distribute the information to the users timely, we will build 3G-based information distribution service, by which the information will be accessed by the 3G-based mobile phone. In the means, the information distribution will help the communication between the users and the information center, which can accept the request and send answer to the users. The information Distribution also makes a plan, and pushes the information to the users' $3 \mathrm{G}$ mobile phone by $3 \mathrm{G}$ network.

\section{Conclusion}

In the paper, we have presented the research, design and analysis of 3GAIMSH hierarchy -- a novel 3G technologies-based agricultural information resource application mobile service hierarchy for rural information services in china. The hierarchy-based application will help integrate distributed agricultural information source into a united environment to be reuse and sharing, and provide the right information to the farmers or other users. In the future, more 3GAIMSH-based applications and tools will be developed, and the paper will study ontology-based agricultural information organization for providing users more intelligent agricultural information service.

\section{Acknowledgements}

The work is supported by the Academy of Science and Technology for Development fund project "intelligent search-based Tibet science \& technology resource sharing technology", Special Project on of The National Department of Science and Technology "TD-SCDMA based application development and demonstration validation in agriculture informationization", Special fund project for Basic Science Research Business Fee, AIIS (No.2009J-06) and the National Science and Technology Program (Grant No. 2006BAD10A06).

\section{References}

Parikh, T.S.: Using Mobile Phones for Secure, Distributed Document Processing in the Developing World. IEEE Pervasive Computing, 4(2), 74-81 (2005)

The real digital divide. The Economist (March 2005) 
Buchanan, G., Farrant, S., Jones, M., Thimbleby, H., Marsden, G., Pazzani, M.: Improving mobile Internet usability. In: WWW 2001: Proceedings of the 10th international conference on World Wide Web, pp. 673-680. ACM Press, New York (2001)

Parikh, T.S., Lazowska, E.D.: Designing an architecture for delivering mobile information services to the rural developing world. In: Proc. WWW 2006 (2006) 